\title{
Erfahrungen der Max Havelaar-Stiftung beim Vertrieb von Produkten aus Entwicklungsländern
}

Monika Schmutz Cattaneo and Rolf Buser

\section{(2) OpenEdition}

12 Journals

Electronic version

URL: http://journals.openedition.org/sjep/1346

DOI: $10.4000 /$ sjep.1346

ISSN: 1663-9677

Publisher

Institut de hautes études internationales et du développement

Printed version

Date of publication: 1 mars 1996

Number of pages: 259-266

ISSN: 1660-5926

\section{Electronic reference}

Monika Schmutz Cattaneo und Rolf Buser, «Erfahrungen der Max Havelaar-Stiftung beim Vertrieb von Produkten aus Entwicklungsländern », Schweizerisches Jahrbuch für Entwicklungspolitik [Online], 15 | 1996, Online erschienen am: 17 Mai 2013, abgerufen am 08 September 2020. URL : http:// journals.openedition.org/sjep/1346; DOI : https://doi.org/10.4000/sjep.1346 


\title{
Erfahrungen der Max Havelaar-Stiftung beim Vertrieb von Produkten aus Entwicklungsländern
}

\author{
Monika Schmutz Cattaneo und Rolf Buser
}

Nach längerer Vorbereitung gründeten im Februar 1992 die sechs grossen Schweizer Hilfswerke Brot für alle, Caritas, Fastenopfer, HEKS, Helvetas und Swissaid die Max Havelaar-Stiftung (Schweiz) nach dem Vorbild der bereits seit 1988 in Holland mit Erfolg tätigen gleichnamigen Stiftung. ${ }^{1}$

Max Havelaar ist der Titel des 1860 erschienenen, autobiographischen Romans des holländischen Schriftstellers Eduard Douwes Dekker, der als Kolonialbeamter in niederländisch Ostindien (heutiges Indonesien) lebte und als einer der ersten die Missstände in den europäischen Kolonien anklagte. Dekker kommt in seinem Roman auch auf die Ausbeutung der einheimischen Arbeitskräfte in den Kaffeeplantagen zu sprechen, und es erstaunt daher nicht, dass der Buchtitel dieses couragierten Mannes heute zum Symbol für die Gütesiegel-Initiativen des Fairen Handels geworden ist.

Kaffee war das erste Produkt, welches durch die Max Havelaar-Stiftung ausgezeichnet wurde. Und zwar knapp zwei Monate nachdem diese mit folgendem Stiftungszweck gegründet worden war: „Die Stiftung fördert den existenzsichernden und umweltschonenden Handel mit benachteiligten Produzenten und die Information über damit zusammenhängende Entwicklungsfragen. Sie vergibt namentlich ein Label zur Auszeichnung von Produkten aus Entwicklungsgebieten, welche Minimalbedingungen in sozialer und ökologischer Hinsicht genügen. Die Stiftung ist nicht gewinnorientiert." Die Max Havelaar-Stiftung treibt selber auch keinen Handel, sondern vermittelt vielmehr zwischen dem Handel in der Schweiz und den Produzentenorganisationen im Süden. Diesen soll der direkte Zugang zum 
Schweizer Markt ermöglicht werden, und zwar über die Kanäle des traditionellen alternativen Handels hinaus. Die Stiftung vergibt gegen die Entrichtung einer Gebühr Lizenzen. Sie schliesst mit interessierten Kreisen aus der Wirtschaft einen Lizenzvertrag ab, der die Bedingungen für den Import der Produkte sowie die Verwendung des Gütesiegels regelt. In diesem Sinne ist die Max Havelaar-Stiftung Zertifizierungs- und Kontrollinstanz.

Die Max Havelaar-Stiftung wird vor allem durch ihre Gründerorganisationen, durch die Beiträge des BAWI und die Einnahmen aus den Lizenzvergaben finanziert. Die Gründerorganisationen haben 1992 141'000 Franken, 1993 321'000 Franken und 1994 306'000 Franken gezahlt. Die Beiträge des BAWI betrugen 1992 485'000 Franken, 1993 321'000 Franken und 1994 305'000 Franken. Die Lizenzeinnahmen beliefen sich 1992 auf 303'000 Franken, 1993 auf 424'000 Franken und 1994 auf 457'000 Franken (Zahlenangaben der Jahresberichte).

Dank den solidarisch und verantwortungsbewusst handelnden Konsumentinnen und Konsumenten, den drei Grossverteilern Coop, Migros und Volg, den Kaffeeröstern und Importeuren der ersten Stunde und der langjährigen Bewusstseinsbildung durch den traditionellen Alternativhandel (Weltladenbewegung), erreichte der faire Kaffee im Detailhandel schon im ersten Jahr einen Marktanteil von 5 Prozent. Dies übertraf selbst die kühnsten Erwartungen sowohl des Handels als auch der Initianten und machte deutlich, dass immer mehr Menschen im Norden die Bereitschaft haben, Entwicklungspolitik und Armutsbekämpfung mit dem Einkaufskorb zu leisten; vorausgesetzt die Qualität stimmt, und die Garantie der Mehrpreisüberführung wird gewährleistet.

\section{Europaweite Zusammenarbeit}

Der beachtliche Erfolg der Max Havelaar-Stiftungen in der Schweiz, den Niederlanden und Belgien führte in weiteren europäischen Ländern zur Gründung von Fair-Handels-Initiativen. Mit diesen gleichgesinnten Organisationen, welche es bis dato in Belgien, Dänemark, Deutschland, Frankreich, Grossbritannien, Holland, Italien, Luxemburg, Oesterreich und gar in Kanada, den USA und Japan gibt, arbeitet Max Havelaar-Schweiz intensiv zusammen. Eine formelle Zusammenarbeit besteht seit 1993. Damals verpflichteten sich die dazumals erst sieben europäischen Gütesiegel-Initiativen des Fairen Handels in einem Vertrag zur Kooperation im Bereich Kaffee. Im Laufe des Jahres 1994 wurden dementsprechende Verträge für weitere Produkte unterzeichnet.

In ihren Abmachungen verpflichten sich die Gütesiegel-Organisationen zu:

- gleichen Auswahlkriterien,

- einer gemeinsamen Kommission, welche die Produzenten auswählt ,

- gleichen Mindestpreisen und Handelsbedingungen,

- einem gemeinsamen Produzentenregister,

- gleichen Monitoring-Grundlagen, 
- einer zentralen Verwaltung der Produzentenregister mit zentraler Datenbank,

- kostensparender Arbeitsteilung.

Im Rahmen dieser Arbeitsteilung ist Max Havelaar-Schweiz für die in der staatlichen sowie privaten schweizerischen Entwicklungshilfe relativ stark berücksichtigten Andenländer (Bolivien, Peru, Ecuador, Kolumbien und Venezuela) zuständig. Die Stiftung begleitet und kontrolliert die dortigen Kaffeeproduzenten und prüft die neuen Anträge von Produzenten aus dieser Region. Darüber hinaus betreute Max Havelaar-Schweiz bis Mitte 1995 das internationale Honigregister sowie die Kontrollen der Imker-Organisationen in Lateinamerika. Die Verwaltung des Kakaoregisters obliegt ebenfalls der Schweizer Geschäftsstelle in Basel.

\section{Honig, Kakao und Zucker mit Gütesiegel}

Bestärkt durch die Verkaufserfolge mit dem fairen Kaffee, lancierte die Max Havelaar-Stiftung im Herbst 1993 inr zweites Produkt: Honig. Neben Caritas und OS3 - mit ihrem schon bestehenden, breiten Angebot an fairem Honig war der Grossverteiler Coop mit einem Honig aus Mittelamerika mit von der Partie. 1994 erzielten die Honig-Lizenznehmer von Max Havelaar ein Verkaufsvolumen von 228'000 Kilogramm. Dies entspricht etwas mehr als 5 Prozent des Importhonigs. Vom gesamten Honigverbrauch in der Schweiz (durchschnittlich 6000 Tonnen jährlich, d.h. über ein Kilo Honig pro Kopf und Jahr) werden je nach inländischer Produktion etwa 50 bis $70 \%$ importiert.

Kaffee und Honig werden zu Fair Trade-Bedingungen hauptsächlich aus dem lateinamerikanischen Raum importiert. Mit dem dritten durch Havelaar ausgezeichneten Produkt, der Schokolade, änderte sich dies. Der Kakao stammt vorwiegend aus Afrika, namentlich aus Ghana, Kamerun und dem vom Bürgerkrieg zerrütteten Sierra Leone. Doch auch die bolivianische Genossenschaft El Ceibo, langjährige Partnerin des traditionellen Alternativen Handels und Lieferantin von biologisch zertifiziertem Kakao, wurde ins Produzentenregister aufgenommen. Ebenso eine Kooperative aus Equador. Die 14 Max Havelaar-Schokoladen erfüllen eine weitere langjährige entwicklungspolitische Forderung der Hilfswerke: sie werden aus fair gehandeltem und im Ursprungsland raffiniertem Rohrzucker hergestellt. Das Schweizer Symbolprodukt Schokolade wurde in seiner neuen Form (fair gehandelter Kakao + fair gehandelter Rohrzucker + Schweizer Milch) im November 1994 der Öffentlichkeit vorgestellt. Das Echo war vielversprechend, war doch neben Coop, Migros und OS3 auch der Schokoladenpionier Maestrani unter den Lizenznehmern. Nach einem Jahr hat sich ein Marktanteil von ca. einem Prozent, resp. 400 Tonnen (entspricht 4 Millionen Tafeln), eingespielt. Drei Hauptgründe können für diesen - im Vergleich zu den anderen Produkten - eher niedrigen Marktanteil angeführt werden: 1. Das Schokoladensortiment ist ums mehrfache breiter als das Kaffeesortiment (pro Grossverteiler ca. 50 Schokoladentafeln). 2. Schokolade ist ein Genussmittel, 
das mit einer hohen Markentreue verbunden ist. 3. Die Grossverteiler Migros und Coop boten zu Beginn nur 1 resp. 2 Tafeln an.

Die Max Havelaar-Bedingungen für den Import von Kaffee, Honig, Kakao und Zucker sind auf die Probleme und Strukturen von Kleinbauern und deren Genossenschaften zugeschnitten. Im Lizenzvertrag, der zwischen der Stiftung und dem Lizenznehmer abgeschlossen wird, sind diese Bedingungen genauestens definiert. Hier einige Beispiele für diese Handelskriterien anhand des Produktes Kaffee:

1. Der Kaffee stammt von ausgewählten Kleinbauern-Genossenschaften (der oft ausbeuterische lokale Zwischenhandel wird ausgeschaltet).

2. Die Abnahme einer festgelegten Menge Kaffee wird garantiert und die Ernte teilweise vorfinanziert.

3. Der Kaffee wird möglichst umweltschonend angebaut und verarbeitet.

4. Die Kaffeebauern erhalten einen existenzsichernden Preis. (D.h.: Der Max Havelaar-Mindestpreis liegt bei $1.26 \mathrm{US} \$ / \mathrm{lb}$ für herkömmlichen und 1.41 US\$/lb für biologisch zertifizierten Kaffee. Befindet sich die Weltmarktnotierung über diesem von Havelaar garantierten Preis, so erhält die Bauernorganisation, resp. der Genossenschaftsverband, ohne Limit nach oben eine zusätzliche Prämie von 5 US\$-cents für herkömmlichen und 15 US\$-cents für organischen Kaffee.)

Erfüllt eine Selbsthilfeorganisation sowohl die entwicklungspolitischen als auch die kommerziellen Auswahlkriterien (Qualität, vertrauenswürdiger Handelspartner) der Max Havelaar-Stiftung, so kommt es zu einem sogenannten Produzentenvertrag. Dieser regelt u.a. folgende Aspekte:

1. Die Produzentenorganisation verpflichtet sich, Anstrengungen zum Abbau von Monokulturen und zur Diversifizierung der Produktion (Vorbeugung Überproduktion) zu unternehmen.

2. Über die Verwendung des Mehrerlöses wird in den Selbsthilfeorganisationen demokratisch entschieden.

3. Die Organisation muss über eine effiziente und transparente Administrationsstruktur sowie Kontrollorgane verfügen.

4. Die Produzentenorganisation muss bereit sein, neue Mitglieder zu akzeptieren und aufzunehmen.

5. Die Selbsthilfeorganisation fördert Dienstleistungen, welche die Autonomie der Organisation und lokale Entwicklungsprozesse stärken (Lagerung, Veredlung, Verpackung, Transport, Kreditwesen, Ausbildung und Gesundheitsvorsorge).

6. Die Organisation bietet ihren Mitgliedern zinsgünstige Kredite an.

\section{Konzeptausweitung}

Mit der Zertifizierung des vierten Produktes waren besonders intensive Vorarbeiten verbunden, denn mit Tee wurde nicht einfach ein weiteres klassisches Kolonialprodukt zu fairen Bedingungen im Markt eingeführt, sondern das be- 
stehende Havelaar-Konzept musste ausgeweitet werden. Tee ist in einer für den europäischen Markt akzeptablen Qualität nur in sehr geringen Mengen aus kleinbäuerlicher Produktion verfügbar. Dies nicht nur deshalb, weil die britischen Kolonialherren den Tee auf grossen Plantagen in Indien, im heutigen Sri Lanka und in Ostafrika anbauten und diese Strukturen nach der Unabhängigkeit der Länder von diesen übernommen wurden, sondern weil Tee unmittelbar nach dem Pflücken verarbeitet werden muss. Dies bedingt namhafte Investitionen in Teefabriken, in denen der Tee bis zur Exportqualität weiterverarbeitet und verpackt wird. Nur Plantagen mit hohen Produktionsvolumen verfügen über diese nötige Infrastruktur, deren Anschaffung für Kleinbauern zu kostspielig ist.

Die Ausweitung des Havelaar-Konzeptes auf Plantagenprodukte hat den Grundsatz des Fairen Handels nicht tangiert. Nach wie vor sollen diejenigen profitieren, welche die Arbeit verrichten. Und dies sind beim Tee die Pflückerinnen und Arbeiter, in der Regel Angestellte der Plantagenbesitzer. Beim Kauf von Max Havelaar Tee wird ein Mehrpreis bezahlt, der in einen Fonds fliesst (der Mehrpreis beträgt 2.50 DM pro Kilo Tee, resp. $3 \mathrm{DM}$ für Tee aus kontrolliert biologischem Anbau). Die Gelder aus diesem Fonds dürfen ausschliesslich zur Verbesserung der Arbeits- und Lebensbedingungen der Plantagenbelegschaft und ihrer Angehörigen verwendet werden. Welche Massnahmen finanziert werden, entscheidet ein aus Arbeitnehmer- und Arbeitgebervertreterlnnen besetztes Komitee. Die Betroffenen wissen selbst am besten, wie sie den Max Havelaar-Mehrerlös am sinnvollsten verwenden: ob beispielsweise zum Kauf von Milchkühen, zur Anschaffung von Spielgeräten für die Kinderkrippen, zur Elektrifizierung der Unterkünfte, zum Bau von Gemeinschaftshäusern, für die Ausbildung der Arbeiterkinder in anderen Berufen oder für Umweltprojekte.

Damit eine Plantage von Max Havelaar anerkennt wird, muss sie eine Reihe von Voraussetzungen erfüllen. So muss sie sich u.a. vertraglich dazu verpflichten, alle im Anbauland geltenden gesetzlichen und tariflichen Mindeststandards (gemäss ILO) einzuhalten und in allen Bereichen absolute Transparenz zu gewährleisten. Die Einhaltung der Havelaar-Bedingungen wird in regelmässigen Abständen vor Ort überprüft. Wie bei den anderen Produkten auch, führt die Havelaar-Stiftung (Schweiz) auch beim Tee gemeinsam mit weiteren europäischen Fair-Handels-Initiativen ein Produzentenregister, in welches bis anhin Teegärten aus Indien, Sri Lanka, Nepal und Zimbabwe aufgenommen wurden (über ein Drittel dieser Gärten liefern biologisch zertifizierten Tee). Die Lizenznehmer für Havelaar-Tee in der Schweiz - Coop, Migros, OS3, Morga, Milliquet und kleinere Anbieter importieren bisher aus Indien und Sri Lanka.

Somit wurde nach Lateinamerika (Kaffee, Kakao, Honig und Zucker) und Afrika (Kaffee und Kakao) erstmals auch Asien in grösserem Rahmen in das Havelaar-Konzept eingebunden (bis anhin wurde nur Zucker aus den Philippinen importiert). 


\section{Organisationen in drei Kontinenten}

Über 100 '000 Kleinbauernfamilien weltweit profitieren vom Fairen Handel in Europa, der jährlich bei den Produzenten ein Exportvolumen von weit über 30 Millionen US\$ auslöst. Alleine aus der Schweiz wurden seit der Gründung der Max Havelaar-Stiftung 8 Millionen Franken Mehrerlös an die Produzenten im Süden ausbezahlt.

Genauer: An 70 Kaffeebauern-Selbsthilfeorganisationen in Bolivien, Brasilien, Costa Rica, der Dominikanischen Republik, El Salvador, Guatemala, Haiti, Honduras, Kamerun, Kolumbien, Mexiko, Nicaragua, Peru, Sierra Leone, Tanzania, Uganda, Venezuela und Zaire, an 9 HonigproduzentenKooperativen in Chile, Guatemala, Mexiko und Uruguay, an 5 Zusammenschlüsse von Kakaobauern in Bolivien, Equador, Ghana, Kamerun und Sierra Leone, an die 4 Zuckerproduzenten in Costa Rica und auf den Philippinen und an die 7 Teegärten in Indien und Sri Lanka.

\section{Qualitative Ergebnisse}

Der Erfolg von Max Havelaar lässt sich nicht nur quantitativ, sondern auch qualitiativ ausweisen. Nebst der quantitativen Einkommensverbesserung bei den Produzenten können folgende qualitativ positiven Auswirkungen festgestellt werden:

1. Das Fair-Handels-Konzept hat sich auch in der Hochpreisphase des Kaffeeweltmarktes behauptet, und zwar sowohl produzenten- als auch marktseitig.

Was die Produzenten anbelangt, so ist noch klarer geworden, dass fairer Handel mehr bedeutet als Mehrpreis. Der Vorfinanzierungsklausel kommt eine ebenso zentrale Bedeutung zu, denn verfügt eine Genossenschaft über keine flüssigen Mittel, um den Kaffee ihrer Mitglieder aufzukaufen, kommt es zu einem Preiszerfall. Dieser kann Auswirkungen auf ganze Regionen haben. In diesem Sinne nehmen die Selbsthilfeorganisationen lokal und regional eine preisregulierende Funktion ein. Es erstaunt daher nicht, dass bei Max Havelaar laufend neue Gesuche zur Aufnahme ins Produzentenregister eingehen. Marktseitig hat sich gezeigt, dass die gestiegenen Weltmarktpreise keinerlei Rückgänge der Käufe von Max Havelaar-Kaffee zur Folge hatten. Dies obwohl gemäss einer repräsentativen Umfrage in der Schweiz $70 \%$ der Konsumentinnen und Konsumenten über den Preisanstieg informiert waren.

2. Max Havelaar nimmt eine Marktöffner-Funktion für Produzenten wahr. Die direkten Handelskontakte der Produzentenorganisationen zu den europäischen Importeuren führen nicht nur zu Verträgen unter Max Havelaar-Bedingungen, sondern ermöglichen auch Verträge zu normalen Marktbedingungen. Diese umgehen den lokalen, ausbeuterischen Zwischenhandel ebenso. 
3. Aufgrund der Mindestpreisgarantie erheben die Importeure und Röster Anspruch auf erstklassige Qualität. Diese Stimulation zur Qualitätsverbesserung (anstatt Quantitätssteigerung) führt mikroökonomisch zu besseren Absatzchancen und ist makroökonomisch daher sinnvoll, weil der strukturelle Überschuss auf dem Weltmarkt nicht noch erhöht wird.

4. Durch die Bioprämie und die Anforderung an eine diversifizierte Produktion wird der Umwelt mehr Rechnung getragen. Immer mehr Havelaar-Partner stellen auf biologischen Anbau um und streben die ÖkoZertifizierung an. Grundsätzlich jedoch können kleinbäuerliche Produktionsstrukturen (Mischkulturen) als weit umweltverträglicher angesehen werden als Monokulturen.

5. Ein weiterer - nicht direkt qualifizierbarer, doch langfristig für das veränderte Bewusstsein im Schweizer Markt wichtiger - Punkt ist folgender: Die Einkäufer der grossen Lizenznehmer reisen z.T. erstmals vor Ort und lernen dort eine Realität kennen, die nicht nur starke persönliche Eindrücke hinterlässt, sondern auch Einfluss auf das Unternehmensbewusstsein haben kann.

6. Der Markterfolg von Max Havelaar hat den Fairen Handel „salonfähig“ gemacht und diesem auch in den Medien zum Durchbruch verholfen. Dadurch wurde auch in anderen Branchen eine Dynamik mitausgelöst, die zur Einführung neuer Produkte und Initiativen mit Labeln führte (Bsp: Blumen, DIP (Textilien), STEP (Teppiche)).

\section{Gefahr eines Label-Salates}

Die Gefahr eines Label-Salates und der Verwässerung des Fair-HandelsBegriffes ist von den Gründerhilfswerken der Havelaar-Stiftung erkannt worden.

Um den Konsumenten und Konsumentinnen weiterhin Transparenz zu gewährleisten, haben die Stifterwerke in den "Strategischen Leitlinien“ folgendes festgehalten: "Max Havelaar soll zur führenden Fair-Handels-Labelinstanz werden; im Food wie im Non-Food Bereich“ und "Die Hilfswerke unternehmen alles, um einen Labelsalat in der Schweiz zu verhindern".

Der Begriff "Fairer Handel“ kann selbstverständlich nicht rechtlich geschützt oder monopolisiert werden. Aus entwicklungspolitischer Sicht jedoch ist es sinnvoll und notwendig, dass der Begriff inhaltlich von den Entwicklungsorganisationen geprägt wird, um Verwirrung und Missbrauch zu vermeiden. Diesbezüglich hat der Max Havelaar-Stiftungsrat auch bereits reagiert und ein Papier mit dem Titel „Anforderungen an den Fairen Handel aus der Sicht der Max Havelaar-Stiftung“ verabschiedet. Dieser Anforderungskatalog lässt genügend Raum für produktespezifische Anpassungen.

Zentraler Punkt der ganzen Problematik bleibt die Transparenz. Diese kann von einer kommerziell unabhängigen Zertifizierungsinstanz mit 
Produktelabel (klarer konzeptioneller Unterschied zum Ladenlabel, wie von der kürzlich gegründeten Teppichinitiative STEP angewendet) problemlos gewährleistet werden.

Wenn die eben angesprochenen konzeptionellen und inhaltlichen Unterschiede in der Kommunikation und Öffentlichkeitsarbeit auf allen Ebenen deutlich gemacht werden, dann ist die Gefahr der Verwirrung (die zu einer generellen Skepsis gegenüber dem Fairen Handel führen kann) bei den Konsumierenden zwar nicht eliminiert, jedoch stark vermindert.

\section{Perspektiven}

Die Max Havelaar-Stiftung wird inren Anspruch als Schrittmacher und führende Fair Handels-Labelinstanz ausbauen, und die Glaubwürdigkeit auch bei allen neuen Havelaar-Produkten mit einem hohen Anforderungsstandard festigen.

Im Vordergrund stehen Produkte mit bedeutendem Umsatzpotential, die entwicklungspolitisch relevant und mit einer klar kommunizierbaren Botschaft an die Konsumenten verbunden sind. Der Aufwand der Stiftung (Produkteentwicklung, Produzenten-Monitoring, Marktbearbeitung) muss in einem gesunden Verhältnis zum Ertrag (für Produzenten und Stiftung) stehen.

Bei der Auswahl neuer Produkte spielt denn auch die Kosten- und Arbeitsteilung innerhalb der europäischen Kooperation der Gütesiegel-Initiativen eine gewichtige Rolle. Eurokonformität (Harmonisierung der Fair-Trade- und Produzentenauswahlkriterien) soll bei Max Havelaar - wie bei den bisherigen Produkten Kaffee, Honig, Zucker, Kakao und Tee - auch in Zukunft eine Selbstverständlichkeit sein.

Im Hinblick auf den europäischen Binnenmarkt sollen keinerlei Wettbewerbsverzerrungen durch unterschiedliche nationale GütesiegelAnforderungen (vgl. Öko-Labels) entstehen. Es wird gar mittelfristig auf ein einheitliches europäisches Label hingearbeitet.

\section{Schlussbemerkung}

Der faire Handel, d.h. der Handel mit menschen- und naturgerechten Produkten, ist im Aufwind. Die Vision, wonach der Süd-Nord-Handel das Prinzip der Kostenwahrheit verinnerlicht, bekommt Konturen. Max Havelaar setzt beispielhaft ein Zeichen, doch um die Position im hart umkämpften Markt zu festigen und auszubauen, sind weiterhin grosse Anstrengungen - v.a. in der Marktbearbeitung - nötig. 